\title{
Non-leptonic and rare kaon decays in lattice QCD
}

\section{C.T. Sachrajda*†}

School of Physics and Astronomy, University of Southampton, Southampton SO17 1BJ, UK

E-mail: ctsesoton.ac.uk

I review the research programme of the RBC-UKQCD collaborations in kaon physics, focussing the discussion on physical quantities which had not been computed previously in lattice simulations. I start with a summary of our recent calculation of the amplitude $A_{2}$ for $K \rightarrow(\pi \pi)_{I=2}$ decays at physical masses and kinematics in which we are able to reproduce the experimental result for $\operatorname{Re} A_{2}$ and to determine $\operatorname{Im} A_{2}$ for the first time. The amplitude $A_{0}$ for $K \rightarrow(\pi \pi)_{I=0}$ decays has not yet been calculated at physical kinematics, but I describe our (energy-conserving) calculations with pion masses $m_{\pi} \simeq 420 \mathrm{MeV}$ and $330 \mathrm{MeV}$ at threshold. From these calculations an explanation of the $\Delta I=1 / 2$ rule ( $\operatorname{Re} A_{0} / \operatorname{Re} A_{2} \simeq 22.5$ ) is emerging; in particular we find a significant cancellation between the two contributions to $\operatorname{Re} A_{2}$ contradicting naïve expectations based on the vacuum insertion hypothesis and suppressing $\operatorname{Re} A_{2}$ relative to $\operatorname{Re} A_{0}$. I discuss prospects for the evaluation of long-distance contributions to the $K_{L}-K_{S}$ mass difference $\left(\Delta m_{K}\right)$ and rare kaon decay amplitudes and describe our recent exploratory study of $\Delta m_{K}$.

The 7th International Workshop on Chiral Dynamics,

August 6 -10, 2012

Jefferson Lab, Newport News, Virginia, USA

\footnotetext{
* Speaker.

${ }^{\dagger}$ For the RBC-UKQCD Collaborations
} 


\section{Introduction}

At Chiral Dynamics 2009, I presented a talk on Kaons on the Lattice in which the main topics were: a) a discussion of the chiral behaviour, b) the determination of the $V_{u s}$ CKM matrix element from $K_{\ell 2}$ and $K_{\ell 3}$ decays, c) the evaluation of the $B_{K}$ parameter of neutral kaon mixing as well as d) the prospects for the evaluation of $K \rightarrow \pi \pi$ decays amplitudes. Recent progress in lattice simulations has led to an impressive precision in the evaluation of quantities such as $V_{u s}$ and $B_{K}$ and hence in the corresponding phenomenology. For example, combining the experimental results for the ratio of kaon and pion leptonic widths and the measured semileptonic $K_{\ell 3}$ decay amplitudes with lattice calculations of the ratio of decay constants $f_{K} / f_{\pi}$ and the $K \rightarrow \pi$ vector form-factor, the Flavianet Lattice Averaging Group [1] quote:

$$
\left|V_{u s}\right|=0.2254(9) \quad \text { and } \quad\left|V_{u d}\right|=0.97427(21) .
$$

If in addition we take the value of $\left|V_{u d}\right|=0.97425$ from super-allowed nuclear $\beta$-decays [2] then the constraint on the unitarity of the first row of the CKM matrix is [1]

$$
\left|V_{u d}\right|^{2}+\left|V_{u s}\right|^{2}+\left|V_{u b}\right|^{2}= \begin{cases}1.0000(7) & \text { using lattice semileptonic form factor } f_{+}(0) \\ 0.9999(6) & \text { using lattice ratio of decay constants } f_{K} / f_{\pi}\end{cases}
$$

For the renormalization group invariant $B_{K}$ parameter FLAG quotes [1]: $\hat{B}_{K}=0.738(20)$. For $K \rightarrow \pi \pi$ decays in 2009 I discussed the prospects for the evaluation of the amplitudes.

At this conference we are hearing about the impressive progress in the precision of lattice calculations of non-perturbative QCD effects in many flavour physics processes and in hadronic structure. It now becomes both possible and necessary to extend the range of physical quantities being studied. In this talk I will review the research programme of the RBC-UKQCD collaboration in kaon physics, focussing on physical quantities which have not been computed previously in lattice simulations. I start with a review of our recent calculation of $A_{2}$, the $K \rightarrow(\pi \pi)_{I=2}$ decay amplitude (where $I$ is the isospin) [3,4]. The calculation is performed at (almost) physical kinematics and the results are presented in Eqs. (2.5) and (2.6) below. We find a value of $\operatorname{Re} A_{2}$ which agrees with the experimental result and determine $\operatorname{Im} A_{2}$ for the first time. The corresponding amplitude with an $I=0$ final state has not yet been evaluated but in Sec. 3 I report on our calculations for pions of masses 330 and $420 \mathrm{MeV}$ at threshold (i.e. with each of the two pions at rest) $[5,6]$. The final two topics, the $K_{L}-K_{S}$ mass difference discussed in Sec.4, and rare kaon decays, discussed in Sec. 5, require the evaluation of long-distance effects represented by matrix elements of the space-time integral of the time-ordered product of two composite operators (rather than the standard calculation of the matrix elements of local operators). Finally I present a brief summary.

\section{2. $K \rightarrow(\pi \pi)_{I=2}$ decay amplitudes}

Before discussing the direct evaluation of $A_{2}$, I need to introduce the ensembles which we use. We have three datasets with $N_{f}=2+1$ Domain Wall Fermions (DWF), two with the Iwasaki gauge action [7-9] and one with the Iwasaki+"Dislocation Suppressing Determinant Ratio" (IDSDR) gauge action [10]. The properties of the actions and datasets can be found in these papers together 


\begin{tabular}{|c|c|c|c|c|c|c|}
\hline units & $m_{\pi}$ & $m_{K}$ & $E_{\pi, 2}$ & $E_{\pi \pi, 0}$ & $E_{\pi \pi, 2}$ & $m_{K}-E_{\pi \pi, 2}$ \\
\hline lattice & $0.1042(2)$ & $0.3707(7)$ & $0.1739(9)$ & $0.2100(4)$ & $0.356(2)$ & $0.015(2)$ \\
$\mathrm{MeV}$ & $142.1(9)$ & $505.5(3.4)$ & $237(2)$ & $286(2)$ & $486(4)$ & $20.0(3.1)$ \\
\hline
\end{tabular}

Table 1: The masses and energies of the mesons in the evaluation of the $K \rightarrow(\pi \pi)_{I=2}$ decay amplitudes. The subscripts 0,2 refer to the cases with each pion's momentum $\left|p_{\pi}\right|$ being 0 or $\sqrt{2} \pi / L$ respectively.

with references to the original theoretical papers. For $K \rightarrow \pi \pi$ decays at physical kinematics the light pions propagate across long distances and hence we require a large physical volume and therefore, in practice, a coarse lattice. We evaluate the matrix elements on a $32^{3}$ spatial lattice with the IDSDR gauge action and a lattice spacing $a=0.14 \mathrm{fm}$ so that the spatial extent of the lattice is approximately $L=4.58 \mathrm{fm}$ in each direction. The unitary pions have masses of about 170 and $250 \mathrm{MeV}$ and the matrix elements are evaluated with partially quenched pions with masses of about $142 \mathrm{MeV}$. The masses and energies are given in Tab. 1.

The amplitude $A_{2}$ is given in terms of the $K \rightarrow \pi \pi$ matrix elements of the following operators:

$$
\begin{aligned}
O_{(27,1)}^{3 / 2} & =\left(\bar{s}^{i} d^{i}\right)_{L}\left\{\left(\bar{u}^{j} u^{j}\right)_{L}-\left(\bar{d}^{j} d^{j}\right)_{L}\right\}+\left(\bar{s}^{i} u^{i}\right)_{L}\left(\bar{u}^{j} d^{j}\right)_{L} \\
O_{7}^{3 / 2} & =\left(\bar{s}^{i} d^{i}\right)_{L}\left\{\left(\bar{u}^{j} u^{j}\right)_{R}-\left(\bar{d}^{j} d^{j}\right)_{R}\right\}+\left(\bar{s}^{i} u^{i}\right)_{L}\left(\bar{u}^{j} d^{j}\right)_{R} \\
O_{8}^{3 / 2} & =\left(\bar{s}^{i} d^{j}\right)_{L}\left\{\left(\bar{u}^{j} u^{i}\right)_{R}-\left(\bar{d}^{j} d^{i}\right)_{R}\right\}+\left(\bar{s}^{i} u^{j}\right)_{L}\left(\bar{u}^{j} d^{i}\right)_{R},
\end{aligned}
$$

where $(27,1)$ denotes the representation of $\mathrm{SU}(3)_{L} \times \mathrm{SU}(3)_{R}$ under which the operator transforms and the labels 7,8 are standard notation for the Electroweak Penguin operators (which transform as the $(8,8)$ representation). The calculation is simplified very significantly by the use of the WignerEckart Theorem:

$$
{ }_{I=2}\left\langle\pi^{+}\left(p_{1}\right) \pi^{0}\left(p_{2}\right)\left|O_{1 / 2}^{3 / 2}\right| K^{+}\right\rangle=\frac{\sqrt{3}}{2}\left\langle\pi^{+}\left(p_{1}\right) \pi^{+}\left(p_{2}\right)\left|O_{3 / 2}^{3 / 2}\right| K^{+}\right\rangle,
$$

where the labels on the operators denote the change in the total isospin $\Delta I$ (superscript) and its third component $\Delta I_{Z}$ (subscript). The flavour structure of the operators $O_{3 / 2}^{3 / 2}$ is $(\bar{s} d)(\bar{u} d)$, compared to $(\bar{s} d)((\bar{u} u)-(\bar{d} d))+(\bar{s} u)(\bar{u} d)$ for the operators $O_{1 / 2}^{3 / 2}$ in Eqs.(2.1)-(2.3). By evaluating the $K^{+} \rightarrow \pi^{+} \pi^{+}$matrix elements on the right-hand side of (2.4), and imposing antiperiodic boundary conditions on one or more components of the $d$-quark and periodic boundary conditions on the $u$-quark, we avoid the necessity of having to isolate an excited state [11,12]. In particular by imposing antiperiodic boundary conditions for the $d$-quark in two directions, the $\pi \pi$ ground state is $\left\langle\pi^{+}(\pi / L, \pi / L, 0) \pi^{+}(-\pi / L,-\pi / L, 0)\right) \mid$ and there is no state with each pion at rest. With the parameters of the simulation this choice of $\left|p_{\pi^{+}}\right|=\sqrt{2} \pi / L$ corresponds closely to a physical decay.

For a detailed description of the calculation, including the renormalization of the operators and the evaluation of finite-volume effects, see [4]. Our final results for the amplitude $A_{2}$ are:

$$
\begin{aligned}
& \operatorname{Re} A_{2}=\left(1.381 \pm 0.046_{\text {stat }} \pm 0.258_{\text {syst }}\right) 10^{-8} \mathrm{GeV} \\
& \operatorname{Im} A_{2}=-\left(6.54 \pm 0.46_{\text {stat }} \pm 1.20_{\text {syst }}\right) 10^{-13} \mathrm{GeV} .
\end{aligned}
$$

The error budget is explained in detail in [4] and is summarised in Tab. 2. The dominant error is due to lattice artefacts since the calculation was performed with a single, rather course, lattice 


\begin{tabular}{|c|c|c|}
\hline Source & $\operatorname{Re} A_{2}$ & $\operatorname{Im} A_{2}$ \\
\hline lattice artefacts & $15 \%$ & $15 \%$ \\
finite-volume corrections & $6.0 \%$ & $6.5 \%$ \\
partial quenching & $3.5 \%$ & $1.7 \%$ \\
renormalization & $1.8 \%$ & $5.6 \%$ \\
unphysical kinematics & $0.4 \%$ & $0.8 \%$ \\
derivative of the phase shift & $0.97 \%$ & $0.97 \%$ \\
Wilson coefficients & $6.6 \%$ & $6.6 \%$ \\
\hline Total & $18 \%$ & $19 \%$ \\
\hline
\end{tabular}

Table 2: Estimated systematic errors from each source to the evaluation of $A_{2}$.

spacing. It will (almost) be eliminated when the calculation is repeated at a second lattice spacing. The current $15 \%$ estimate, intended to be conservative, is obtained by (i) studying the dependence on $a$ of quantities which have been calculated at several lattice spacings including the one used for this calculation, and in particular (ii) by determining the $a$ dependence of $B_{K}$, which is also given by the matrix element of a $(27,1)$ operator.

The result for $\operatorname{Re} A_{2}$ in (2.5) agrees well with the experimental value of $1.479(4) \times 10^{-8} \mathrm{GeV}$ obtained from $K^{+}$decays and $1.573(57) \times 10^{-8} \mathrm{GeV}$ obtained from $K_{S}$ decays. $\operatorname{Im} A_{2}$ is unknown so that our result provides its first direct determination. For the phase of $A_{2}$ we find $\operatorname{Im} A_{2} / \operatorname{Re} A_{2}=$ $-4.42(31)_{\text {stat }}(89)_{\text {syst }} 10^{-5}$. We can combine our result for $\operatorname{Im} A_{2}$ with the experimental values of $\operatorname{Re} A_{2}, \operatorname{Re} A_{0}=3.3201(18) 10^{-7} \mathrm{GeV}$ and $\varepsilon^{\prime} / \varepsilon$ to obtain:

$$
\frac{\operatorname{Im} A_{0}}{\operatorname{Re} A_{0}}=-1.61(19)_{\text {stat }}(20)_{\text {syst }} \times 10^{-4} .
$$

Of course, we wish to confirm this value directly and are working to achieve this.

Finally we present the contributions to $A_{2}$ from the three operators renormalised in the $\overline{\mathrm{MS}}$ NDR scheme at a scale of $3 \mathrm{GeV}$ :

$$
\begin{array}{lc|ll}
\operatorname{Re}\left(\mathrm{A}_{2}\right)_{(27,1)} & (1.398 \pm 0.044) 10^{-8} \mathrm{GeV} & \operatorname{Im}\left(\mathrm{A}_{2}\right)_{(27,1)} & (1.55 \pm 0.36) 10^{-13} \mathrm{GeV} \\
\operatorname{Re}\left(\mathrm{A}_{2}\right)_{7} & (4.29 \pm 0.24) 10^{-11} \mathrm{GeV} & \operatorname{Im}\left(\mathrm{A}_{2}\right)_{7} & (4.47 \pm 0.25) 10^{-14} \mathrm{GeV} \\
\operatorname{Re}\left(\mathrm{A}_{2}\right)_{8} & (-2.14 \pm 0.12) 10^{-10} \mathrm{GeV} & \operatorname{Im}\left(\mathrm{A}_{2}\right)_{8} & (-8.14 \pm 0.47) 10^{-13} \mathrm{GeV} .
\end{array}
$$

The dominant contribution to $\operatorname{Re} A_{2}$ comes, as expected, from the $(27,1)$ operator and to $\operatorname{Im} A_{2}$ from the EWP operator $O_{8}^{3 / 2}$ (but with a non-negligible contribution from $O_{(27,1)}^{3 / 2}$ ). More details can be found in [4].

\section{3. $K \rightarrow(\pi \pi)_{I=0}$ decay amplitudes}

In the $I=0$ channel the two pions have vacuum quantum numbers, which is the major obstacle to a precise determination of $A_{0}$. The two-pion contribution to correlation functions whose time ( $\mathrm{t}$ ) behaviour is $\exp \left[-E_{\pi \pi} t\right]$ is obtained after subtracting the constant vacuum contribution leading to a major loss of precision. To illustrate this, consider two-pion correlation functions which are an important ingredient in the evaluation of $A_{0}$ and $A_{2}$. These are given by the four diagrams in Fig.1. 

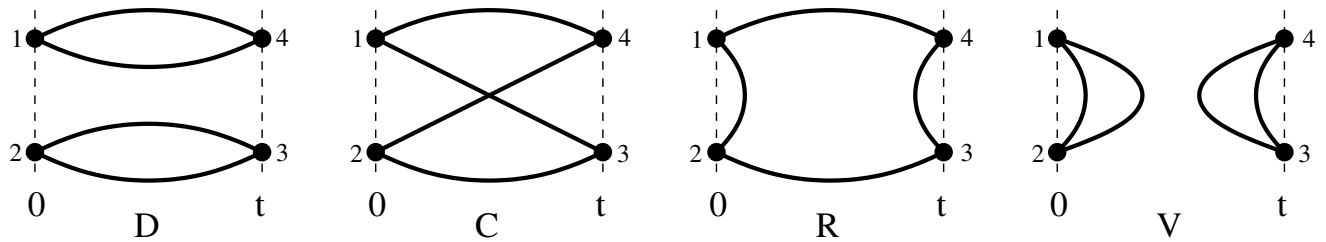

Figure 1: The four contributions to the propagation of a two-pion state.

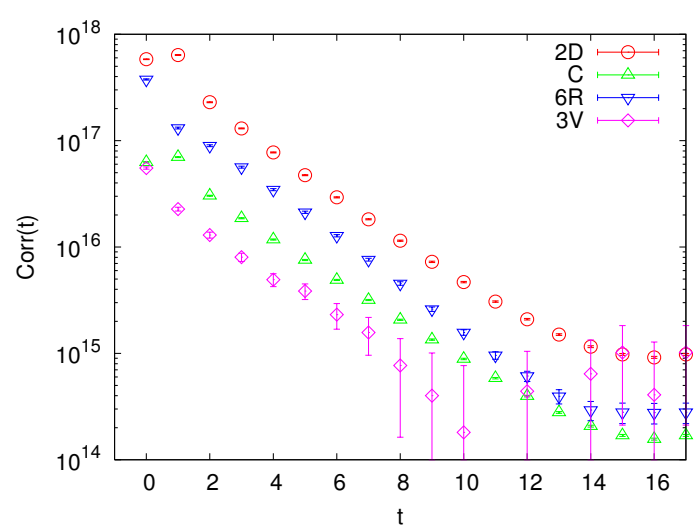

Figure 2: The four contributions to the correlation function of two pions in an $\mathrm{I}=0$ state.

The propagation of an $I=2$ two-pion state is given by the combination $D-C$ whereas the propagation of the $I=0$ state is proportional to the combination $2 \mathrm{D}+\mathrm{C}-6 \mathrm{R}+3 \mathrm{~V}$. In [5] we report on a high-statistics exploratory study of $K \rightarrow \pi \pi$ decays on a $16^{3} \times 32$ Iwasaki lattice with $a^{-1}=$ $1.73 \mathrm{GeV}$ and $m_{\pi} \simeq 420 \mathrm{MeV}$. The propagators were evaluated with sources on each time-slice. Fig. 2 shows the four contributions to the $I=0$ correlation function, illustrating that the error in the $V$ diagram (after the subtraction of the vacuum contribution) is the dominant one [13].

The evaluation of $A_{0}$ requires the calculation of $48 K \rightarrow \pi \pi$ contractions which we classify into the 6 different types in Fig.3. Mix3 and Mix4 are needed to subtract the power ultraviolet divergences which are proportional to matrix elements of the pseudoscalar density $\bar{s} \gamma_{5} d$.

The results from our exploratory calculations for an (almost) on-shell (i.e. energy conserving) decay of a kaon into two $420 \mathrm{MeV}$ pions at rest are:

$$
\begin{array}{ll}
\operatorname{Re} A_{0}=(3.80 \pm 0.82) 10^{-7} \mathrm{GeV} & \operatorname{Im} A_{0}=-(2.5 \pm 2.2) 10^{-11} \mathrm{GeV} \\
\operatorname{Re} A_{2}=(4.911 \pm 0.031) 10^{-8} \mathrm{GeV} & \operatorname{Im} A_{2}=-(5.502 \pm 0.0040) 10^{-13} \mathrm{GeV}
\end{array}
$$

This is an exploratory calculation in which we are learning how to calculate $A_{0}$. In spite of the unphysical kinematics, all the ingredients were fully included. The device of applying anti-periodic boundary conditions to a single quark field used in the evaluation of $A_{2}$ in Sec. 2, cannot be used for $A_{0}$ and hence the calculations described here were performed with the pions at rest. We are developing more sophisticated boundary conditions mixing quarks and anti-quarks and an isospin rotation, the so called G-parity boundary conditions [14], in order to be able to perform calcula- 


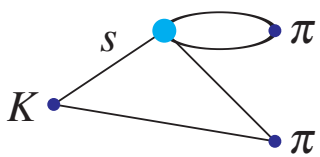

Type1

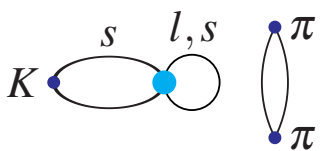

Type4

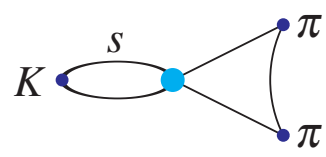

Type2

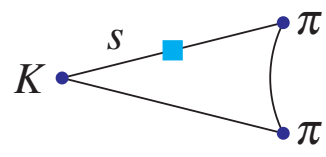

Mix3

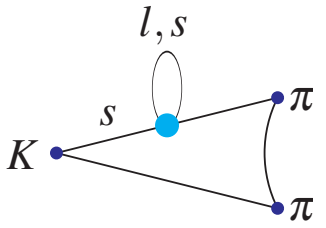

Type3

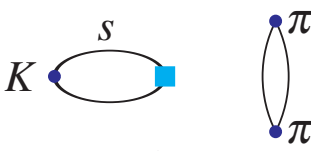

$\operatorname{Mix} 4$

Figure 3: The 6 types of diagram contributing to the correlation functions for the evaluations of $A_{0}$.
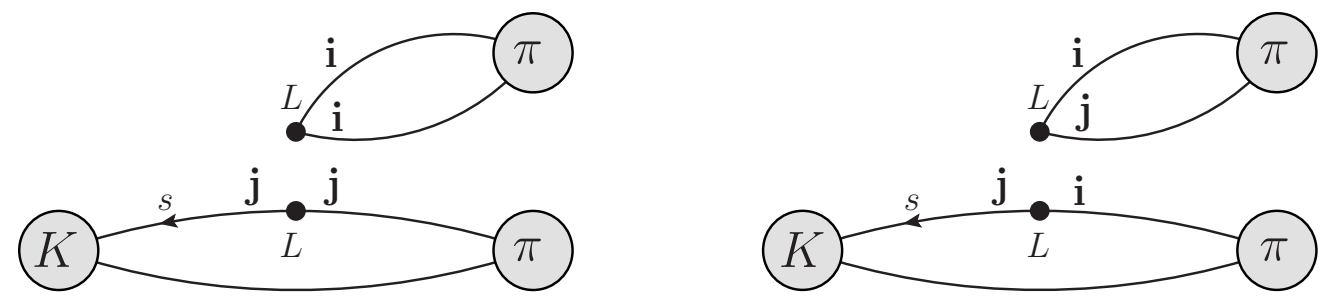

Figure 4: The two contractions (1) and (2) contributing to $\operatorname{Re} A_{2}$. They are distinguished by the colour summation ( $i, j$ denote colour). $s$ denotes the strange quark and $L$ that the currents are left-handed.

tions at physical kinematics. We also continue to develop techniques to enhance the signal and to evaluate disconnected diagrams effectively.

I mention in passing that the evaluation of disconnected diagram has allowed us to study the masses and mixing of the $\eta$ and $\eta^{\prime}$ mesons [15].

\subsection{Emerging understanding of the $\Delta I=1 / 2$ Rule [16]}

Although the calculation of $A_{0}$ described above was performed at unphysical kinematics it nevertheless already shows a significant relative enhancement of $A_{0}$ compared to $A_{2}$. This study has recently been extended to the $24^{3}$ Iwasaki ensembles [7], still with $a^{-1}=1.73 \mathrm{GeV}$ but with $m_{\pi} \simeq 330 \mathrm{MeV}$, and the results for the $\Delta I=1 / 2$ rule can be summarised as:

1. $16^{3} \times 32$ ensembles; $877 \mathrm{MeV}$ kaon decaying into two $422 \mathrm{MeV}$ pions at rest:

$$
\frac{\operatorname{Re} A_{0}}{\operatorname{Re} A_{2}}=9.1(21) \text {. }
$$

2. $24^{3} \times 64$ ensembles; $662 \mathrm{MeV}$ kaon decaying into two $329 \mathrm{MeV}$ pions at rest:

$$
\frac{\operatorname{Re} A_{0}}{\operatorname{Re} A_{2}}=12.0(17) .
$$



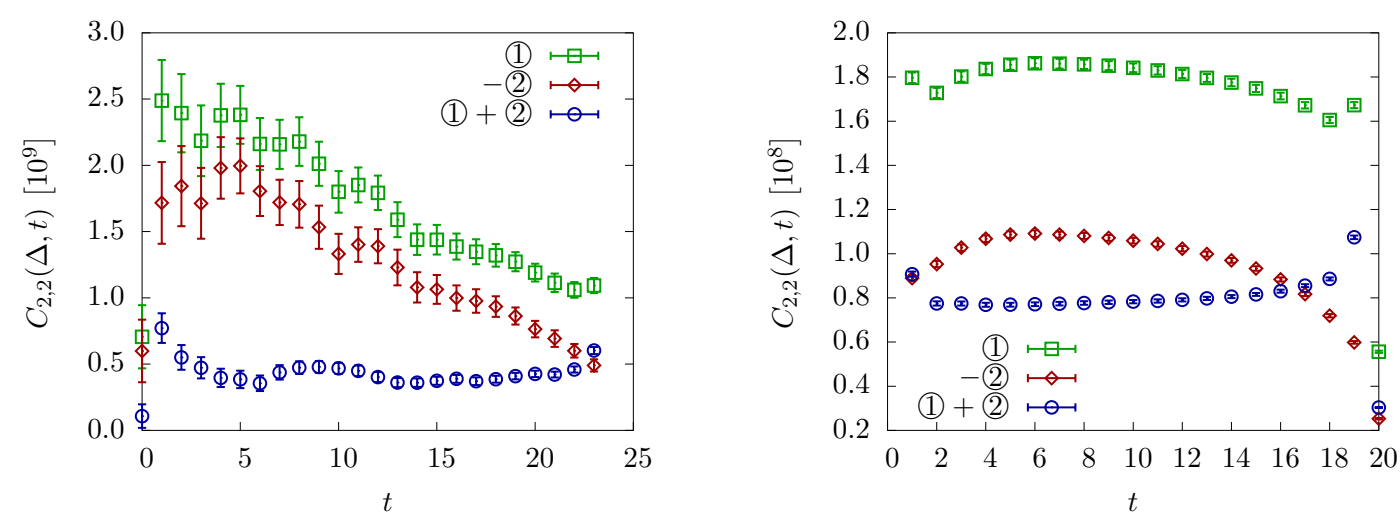

Figure 5: The contractions (1), - (2) and (1) + (2) as functions of $t$. The left hand plot corresponds to the simulation at physical masses and kinematics described in Sec. 2 and the right-hand plot to the $24^{3}$ simulation with $330 \mathrm{MeV}$ pions at rest.

While these results differ significantly from the observed value of 22.5 , because the calculations were not performed at physical kinematics, it is nevertheless interesting to understand the origin of the significant enhancement of the ratios in (3.2) and (3.3). We find that almost all of the contribution to the real parts of $A_{0}$ and $A_{2}$ come from the matrix elements of the current-current operators. The correlation function for $\operatorname{Re} A_{2}$ is simply proportional to the sum (1)+(2) of the two contractions shown in Fig. $4^{1}$. Colour counting (vacuum-insertion hypothesis) suggests that (2) $\approx \frac{1}{3}(1)$ but our calculations show that this is not the case. For both the $16^{3}$ and $24^{3}$ simulations for which $\operatorname{Re} A_{0} / \operatorname{Re} A_{2}$ is given in (3.2) and (3.3) respectively and for the simulation at physical kinematics described in Sec. 2 for which $\operatorname{Re} A_{2}$ is given in (2.5), contractions (1) and (2) have an opposite sign and comparable magnitude. This is illustrated in Fig.5 where the very significant cancellation between (1) and (2) is evident. We note that while most early phenomenology was performed assuming the approximate validity of the vacuum insertion hypothesis, the authors of [17] also found a suppression of $\operatorname{Re} A_{2}$ using the $1 / N$ expansion with a particular ansatz for matching the short and long-distance factors at scales of $0.6-0.8 \mathrm{GeV}$.

No calculation of $A_{0}$ has yet been performed at physical kinematics, but for both the $16^{3}$ and $24^{3}$ simulations the largest contribution comes from the current-current operators. The coefficients of the dominant contractions (1) and (2) now enter with opposite signs leading to an enhancement of $\operatorname{Re} A_{0}$. The largest contribution comes from the operator traditionally labelled $Q_{2}$ for which the correlation function is proportional to 2(1)-(2) whereas that for $Q_{1}$, which is the next largest, is proportional to 2(2)-(1). Both contribute with the same sign to $\operatorname{Re} A_{0}$.

A definitive quantitative explanation of the $\Delta I=1 / 2$ rule awaits the completion of a computation of $\operatorname{Re} A_{0}$ at physical masses and kinematics and the reproduction of the experimental result $\operatorname{Re} A_{0} / \operatorname{Re} A_{2} \simeq 22.5$. Nevertheless we find that the picture which is emerging from our calculations is convincing. It suggests that the origin of the $\Delta I=1 / 2$ rule is a combination of the well-known factor of approximately 2 due to the perturbative running from the electroweak scale to a few $\mathrm{GeV}$, the suppression of $\operatorname{Re} A_{2}$ due to the cancellation in (1)+(2) and a corresponding enhancement in $A_{0}$. We stress that the suppression in $\operatorname{Re} A_{2}$ has been observed in the calculation at physical masses

\footnotetext{
${ }^{1}$ The notation (1), $n=1$ - 48, was introduced in [5] to label the 48 contractions contributing to the calculation of $A_{0}$.
} 


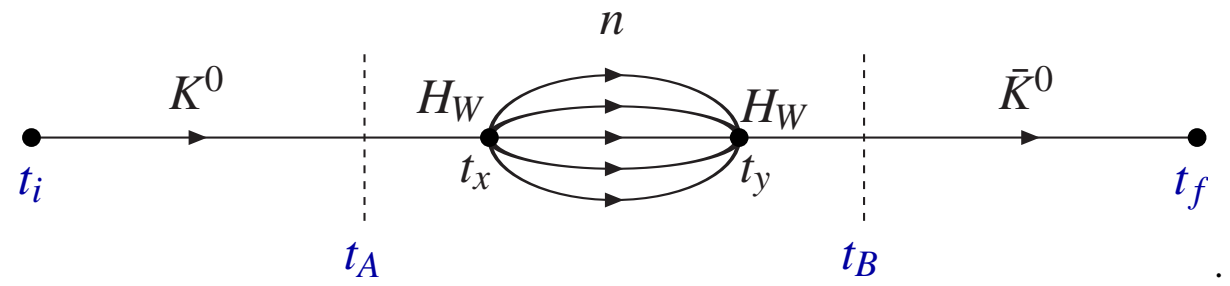

Figure 6: Schematic diagram of the evaluation of long-distance contributions to $\Delta m_{K}$. The integrals in (4.2) are performed over the fiducial volume $t_{A} \leq t_{x, y} \leq t_{B}$. $n$ labels a generic state between the two $H_{W}$.

and kinematics described in Sec.2 [16]. This picture is supported by the observation that $\operatorname{Re} A_{0}$ is found to depend only mildly on the quark masses with the results from the $16^{3}$ and $24^{3}$ simulations already close to the physical value. $\operatorname{Re} A_{2}$ on the other hand depends more strongly on the kinematics, a dependence which we attribute to the strong partial cancellation between (1) and (2).

\section{The $K_{L}-K_{S}$ Mass Difference [18]}

In this and the following section I will discuss our early attempts to extend the range of physical quantities which can be computed in lattice simulations by computing long-distance contributions. These are not given in terms of matrix elements of local operators but require the evaluation of integrals of non-local products of operators such as

$$
\int d^{4} x \int d^{4} y\left\langle h_{2}\left|T\left\{O_{1}(x) O_{2}(y)\right\}\right| h_{1}\right\rangle
$$

where $O_{1,2}$ are local composite operators. For $\Delta m_{K}=m_{K_{L}}-m_{K_{S}}$, the relevant integral is

$$
\int d^{4} x \int d^{4} y\left\langle\bar{K}^{0}\left|T\left\{H_{W}(x) H_{W}(y)\right\}\right| K^{0}\right\rangle,
$$

where $H_{W}$ is the $\Delta S=1$ weak Hamiltonian. In both (4.1) and (4.2) $T$ represents time-ordering.

In a finite volume, the practical way to isolate the initial and final states correctly while still performing the time integrals is to integrate over a large subinterval in time, $t_{A} \leq t_{x, y} \leq t_{B}$, and to create the $K^{0}$ and annihilate the $\bar{K}^{0}$ well outside of this region (see Fig. 6). This is the natural modification of standard field theory for which the asymptotic states are prepared at $t \rightarrow \pm \infty$ and then the operators are integrated over all time. The corresponding 4-point correlation function is

$$
C_{4}\left(t_{A}, t_{B} ; t_{i}, t_{f}\right)=\left|Z_{K}\right|^{2} e^{-m_{K}\left(t_{f}-t_{i}\right)} \sum_{n} \frac{\left\langle\bar{K}^{0}\left|H_{W}\right| n\right\rangle\left\langle n\left|H_{W}\right| K_{0}\right\rangle}{\left(m_{K}-E_{n}\right)^{2}}\left\{e^{\left(M_{K}-E_{n}\right) T}-\left(m_{K}-E_{n}\right) T-1\right\},
$$

where $T=t_{B}-t_{A}+1$ and $Z_{K}$ is the matrix element of the kaon interpolating operator between the vacuum and a kaon at rest. From the coefficient of $T$ we can obtain

$$
\Delta m_{K}^{\mathrm{FV}} \equiv 2 \sum_{n} \frac{\left\langle\bar{K}^{0}\left|\mathscr{H}_{W}\right| n\right\rangle\left\langle n\left|\mathscr{H}_{W}\right| K_{0}\right\rangle}{\left(m_{K}-E_{n}\right)} .
$$

In order to evaluate $\Delta m_{K}$ itself we need to be able to: 


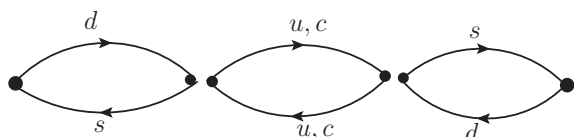

Type 1

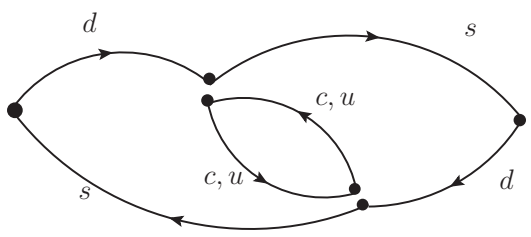

Type 2

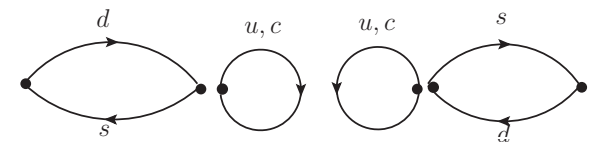

Type 3

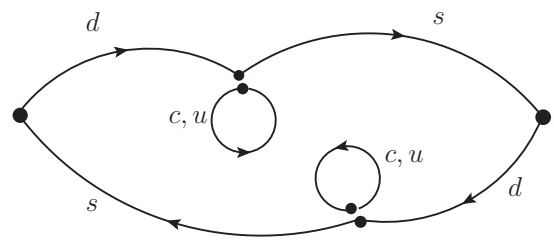

Type 4

Figure 7: Four types of diagram which have to be evaluated for the calculation of $\Delta m_{K}$.

1. Relate $\Delta m_{K}$ and $\Delta m_{K}^{\mathrm{FV}}$. This is an extension of the theory of finite-volume effects for two-pion states including the Lüscher quantization condition and the Lellouch-Lüscher factor $[18,19]$.

2. Control the additional ultraviolet divergences as the weak Hamiltonians come close together. This is facilitated by the GIM mechanism requiring the presence of charm quarks. We find that after the GIM subtraction of the power divergences there are no remaining logarithmic ones [18]. 3. Evaluate the four types of diagram illustrated in Fig. 7.

In our exploratory study on the $16^{3}$ ensembles with $m_{\pi}=420 \mathrm{MeV}$, we only evaluate the type 1 and 2 graphs. The development of techniques necessary to evaluate disconnected diagrams effectively is an area of worldwide active research and in this study we focus instead on learning how to control the remaining systematics. The physical value of $\Delta m_{K}$ is $3.483(6) \times 10^{-12} \mathrm{MeV}$, whereas we obtain values in the range $\{5.81(28)-10.58(75)\} \times 10^{-12} \mathrm{MeV}$ as $m_{K}$ is varied from 563 to $839 \mathrm{MeV}$. In spite of the unphysical masses and neglecting type 3 and 4 diagrams we are nevertheless able to obtain a result reasonably close to the physical value and we are encouraged to proceed to a full physical calculation.

The details of our study are presented in [18]. As an example of the investigations, consider the ultraviolet behaviour of the $Q_{1}-Q_{1}$ correlation function (i.e. taking the $Q_{1}$ component of $H_{W}$ in

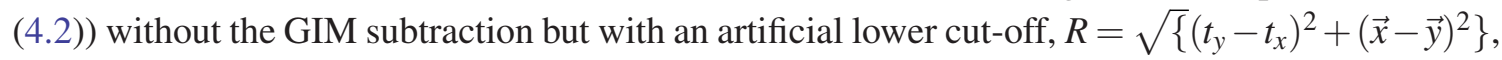
on the separation of the two $Q_{1}$ insertions. The plot in Fig.8 exhibits the quadratic divergence as the two operators come together. This divergence is canceled by the GIM mechanism.

\section{Rare Kaon Decays}

Rare kaon decays which are dominated by short-distance FCNC processes, $K \rightarrow \pi v \bar{v}$ in particular, provide a potentially valuable window on new physics at high-energy scales. The decays $K_{L} \rightarrow \pi^{0} e^{+} e^{-}$and $K_{L} \rightarrow \pi^{0} \mu^{+} \mu^{-}$are also considered promising because the long-distance effects are reasonably under control using ChPT. They are sensitive to different combinations of short- 


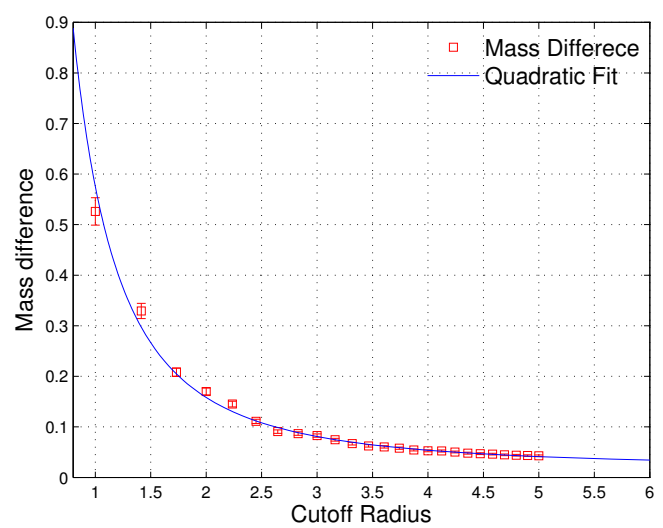

Figure 8: Contribution to the mass difference from the correlation function of $Q_{1}-Q_{1}$ as a function of the cut of radius $R$ as described in the text. The curve is a fit to the function $b / R^{2}+c$, where $b, c$ are constants. The quadratic divergence is cancelled when the GIM mechanism is applied.

distance FCNC effects and hence in principle provide additional discrimination to the neutrino modes. A challenge for the lattice community is therefore either to calculate the long-distance effects reliably or at least to determine the Low Energy Constants of ChPT.

As an example consider the decay $K_{L} \rightarrow \pi^{0} \ell^{+} \ell^{-}$which has three main contributions to the amplitude [20], (i) short distance contributions corresponding to matrix elements of the local operators $\left(\bar{s} \gamma_{\mu} d\right)\left(\bar{\ell} \gamma^{\mu} \ell\right)$ and $\left(\bar{s} \gamma_{\mu} d\right)\left(\bar{\ell} \gamma^{\mu} \gamma_{5} \ell\right)$; (ii) long-distance indirect CP-violating contribution from the CP-even component of $K_{L}, A_{I C P V}\left(K_{L} \rightarrow \pi^{0} \ell^{0} \ell^{-}\right)=\varepsilon A\left(K_{1} \rightarrow \pi^{0} \ell^{+} \ell^{-}\right)$and (iii) the two-photon CPconserving contribution $K_{L} \rightarrow \pi^{0}\left(\gamma^{*} \gamma^{*} \rightarrow \ell^{+} \ell^{-}\right)$. For the corresponding phenomenology see [21]; for example the branching ratios for the $\mathrm{CP}$-violating component are:

$$
\begin{aligned}
\operatorname{Br}\left(K_{L} \rightarrow \pi^{0} e^{+} e^{-}\right)_{\mathrm{CPV}} & =10^{-12} \times\left\{15.7\left|a_{S}\right|^{2} \pm 6.2\left|a_{S}\right|\left(\frac{\operatorname{Im} \lambda_{t}}{10^{-4}}\right)+2.4\left(\frac{\operatorname{Im} \lambda_{t}}{10^{-4}}\right)^{2}\right\} \\
\operatorname{Br}\left(K_{L} \rightarrow \pi^{0} \mu^{+} \mu^{-}\right)_{\mathrm{CPV}} & =10^{-12} \times\left\{3.7\left|a_{S}\right|^{2} \pm 1.6\left|a_{S}\right|\left(\frac{\operatorname{Im} \lambda_{t}}{10^{-4}}\right)+1.0\left(\frac{\operatorname{Im} \lambda_{t}}{10^{-4}}\right)^{2}\right\}
\end{aligned}
$$

where $a_{S}$ is the amplitude for the decay $K_{S} \simeq K_{1} \rightarrow \pi^{0} \ell^{+} \ell^{-} .\left|a_{S}\right|=1.06_{-0.21}^{+0.26}$ but the sign of $a_{S}$ is unknown. One goal of future lattice calculations is the determination of $a_{S}$, together with similar other quantities.

The generic non-local matrix elements which we wish to evaluate are

$$
\begin{aligned}
X & \equiv \int_{-\infty}^{\infty} d t_{x} d^{3} x\left\langle\pi(p)\left|\mathrm{T}\left[J_{\mu}(0) H_{W}(x)\right]\right| K(\overrightarrow{0})\right\rangle \\
& =i \sum_{n} \frac{\left\langle\pi(p)\left|J_{\mu}(0)\right| n\right\rangle\left\langle n\left|H_{W}(0)\right| K(\overrightarrow{0})\right\rangle}{m_{K}-E_{n}+i \varepsilon}-i \sum_{n_{s}} \frac{\left\langle\pi(p)\left|H_{W}(0)\right| n_{s}\right\rangle\left\langle n_{s}\left|J_{\mu}(0)\right| K(\overrightarrow{0})\right\rangle}{E_{n_{s}}-E_{\pi}+i \varepsilon}
\end{aligned}
$$

$J_{\mu}$ represents a vector or axial current and $\{|n\rangle\}$ and $\left\{\left|n_{s}\right\rangle\right\}$ represent complete sets of non-strange 
and strange states. In Euclidean space we envisage calculating correlation functions of the form

$$
C \equiv \int_{-T_{a}}^{T_{b}} d t_{x}\left\langle\phi_{\pi}\left(\vec{p}, t_{\pi}\right) \mathrm{T}\left[J_{\mu}(0) H_{W}\left(t_{x}\right)\right] \phi_{K}^{\dagger}\left(\overrightarrow{0}, t_{K}\right)\right\rangle \equiv \sqrt{Z_{K}} \frac{e^{-m_{K}\left|t_{K}\right|}}{2 m_{K}} X_{E} \sqrt{Z_{\pi}} \frac{e^{-E_{\pi} t_{\pi}}}{2 E_{\pi}}
$$

where $\phi_{\pi}$ and $\phi_{K}$ are interpolating operators and

$$
\begin{aligned}
& X_{E_{-}}=-\sum_{n} \frac{\left\langle\pi(p)\left|J_{\mu}(0)\right| n\right\rangle\left\langle n\left|H_{W}(0)\right| K\right\rangle}{m_{K}-E_{n}}\left(1-e^{\left(m_{K}-E_{n}\right) T_{a}}\right) \quad \text { and } \\
& X_{E_{+}}=\sum_{n_{s}} \frac{\left\langle\pi(p)\left|H_{W}(0)\right| n_{s}\right\rangle\left\langle n_{s}\left|J_{\mu}(0)\right| K\right\rangle}{E_{n_{s}}-E_{\pi}}\left(1-e^{-\left(E_{n_{s}}-E_{\pi}\right) T_{b}}\right) .
\end{aligned}
$$

We use the time dependence to subtract the exponential terms.

The authors of ref. [22] investigated the ultraviolet behaviour as the current $J_{\mu}$ approaches $H_{W}$. Dimensional counting allows for a quadratic divergence but gauge invariance for the vector current suggests that the degree of divergence is reduced by 2 to result in a logarithmic divergence. This was checked in an explicit one-loop perturbative calculation for Wilson and Clover fermion actions in [22]. This absence of power divergences does not require the use of the GIM mechanism and for a chiral symmetric formulation of lattice QCD, such as DWF, the same applies for the axial current.

The primary reason for discussing the prospects for calculations of rare kaon decay amplitudes at this conference is to encourage a discussion with the wider community with the aim of optimising plans for our future research programme at an early stage.

\section{Summary, Conclusions and Prospects}

The goal of the lattice flavour-physics community is to develop a programme with an ever increasing precision and a growing range of physical quantities which can be studied. Precision flavour physics complements the large $p_{\perp}$ approach to testing the limits of the standard model and searches for new physics. Standard quantities, such as quark masses, mesonic decay constants, $B_{K}$ and $K_{\ell 3}$ form factors are now calculated with excellent precision [1]. In this talk I have reviewed recent progress from the RBC-UKQCD collaboration, focussing in particular on new quantities in kaon physics which we are learning to compute.

- We have performed the first direct calculation of the $K \rightarrow(\pi \pi)_{I=2}$ decay amplitude $A_{2}$. The calculation was performed at physical kinematics and the priority now will be to reduce substantially the main source of systematic uncertainty, that due to lattice artefacts, by repeating the calculation at other lattice spacings.

- Although significant technical problems remain, we are well on our way to calculating $A_{0}$. The development of $G$-parity boundary conditions will enable us to perform the calculations at physical kinematics and hence to understand the $\Delta I=1 / 2$ rule quantitatively and to reproduce the experimental value of $\varepsilon^{\prime} / \varepsilon$, both from first principles.

- An explanation of the $\Delta I=1 / 2$ rule is emerging from our calculations. In particular, in contradiction to expectations from the vacuum insertion hypothesis, there is a very significant cancellation between the two contributions to $\operatorname{Re} A_{2}$ and hence an enhancement of $\operatorname{Re} A_{0} / \operatorname{Re} A_{2}$.

- We are beginning to tackle the calculation of long-distance effects in $\Delta m_{K}$ and rare kaon decays. 
Finally I stress most strongly, that as the range of quantities which can be studied in lattice simulations is increased, we will need continued collaboration with both the theoretical and experimental Chiral Dynamics communities to organise our projects most effectively.

Acknowledgements I warmly thank my colleagues from the RBC-UKQCD collaboration with whom the ideas and calculations described in this talk were developed and performed. I acknowledge partial support from STFC Grant ST/G000557/1.

\section{References}

[1] G. Colangelo, S. Durr, A. Juttner, L. Lellouch, H. Leutwyler, V. Lubicz, S. Necco and C. T. Sachrajda et al., Eur. Phys. J. C 71 (2011) 1695 [arXiv:1011.4408 [hep-lat]].

[2] J. C. Hardy and I. S. Towner, Phys. Rev. C 79 (2009) 055502 [arXiv:0812.1202 [nucl-ex]].

[3] T. Blum, P. A. Boyle, N. H. Christ, N. Garron, E. Goode, T. Izubuchi, C. Jung and C. Kelly et al., Phys. Rev. Lett. 108 (2012) 141601 [arXiv:1111.1699 [hep-lat]].

[4] T. Blum, P. A. Boyle, N. H. Christ, N. Garron, E. Goode, T. Izubuchi, C. Jung and C. Kelly et al., Phys. Rev. D 86 (2012) 074513 [arXiv:1206.5142 [hep-lat]].

[5] T. Blum, P. A. Boyle, N. H. Christ, N. Garron, E. Goode, T. Izubuchi, C. Lehner and Q. Liu et al., Phys. Rev. D 84 (2011) 114503 [arXiv:1106.2714 [hep-lat]].

[6] Qi Liu, Ph.D. thesis (2012) Columbia University.

[7] C. Allton et al. [RBC-UKQCD Collaboration], Phys. Rev. D 78 (2008) 114509 [arXiv:0804.0473 [hep-lat]].

[8] C. Allton et al. [RBC and UKQCD Collaboration], Phys. Rev. D 76 (2007) 014504 [hep-lat/0701013].

[9] Y. Aoki et al. [RBC and UKQCD Collaborations], Phys. Rev. D 83 (2011) 074508 [arXiv:1011.0892 [hep-lat]].

[10] R. Arthur et al. [RBC and UKQCD Collaborations], arXiv:1208.4412 [hep-lat].

[11] C. Kim, Nucl. Phys. Proc. Suppl. 129 (2004) 197 [hep-lat/0311003].

[12] C. H. Kim, Nucl. Phys. Proc. Suppl. 140 (2005) 381-383.

[13] Q. Liu [RBC and UKQCD Collaboration], PoS LATTICE 2010 (2010) 314 [arXiv:1010.3768 [hep-lat]].

[14] C. Kim and N. H. Christ, PoS LAT 2009 (2009) 255 [arXiv:0912.2936 [hep-lat]].

[15] N. H. Christ, C. Dawson, T. Izubuchi, C. Jung, Q. Liu, R. D. Mawhinney, C. T. Sachrajda and A. Soni et al., Phys. Rev. Lett. 105 (2010) 241601 [arXiv:1002.2999 [hep-lat]].

[16] P. A. Boyle et al. [RBC and UKQCD Collaborations], arXiv:1212.1474 [hep-lat].

[17] W. A. Bardeen, A. J. Buras and J. M. Gerard, Phys. Lett. B 192 (1987) 138.

[18] N. H. Christ, T. Izubuchi, C. T. Sachrajda, A. Soni and J. Yu, arXiv:1212.5931 [hep-lat].

[19] N. H. Christ, G. Martinelli and C. T. Sachrajda (in preparation).

[20] F. Mescia, C. Smith and S. Trine, JHEP 0608 (2006) 088 [hep-ph/0606081].

[21] V. Cirigliano, G. Ecker, H. Neufeld, A. Pich and J. Portoles, Rev. Mod. Phys. 84 (2012) 399 [arXiv:1107.6001 [hep-ph]].

[22] G. Isidori, G. Martinelli and P. Turchetti, Phys. Lett. B 633 (2006) 75 [hep-lat/0506026]. 